\title{
A novel psychovisual model on an independent video frame for an almost lossless compression
}

\begin{abstract}
A psychoacoustic model is well established in an audio coding. From the beginning of computer audio era since the previous century, an audio coding has already operated on a large one-dimensional fast Fourier transform (FFT) as a basic industrial coding. This paper investigates a quantitative experimental impact on almost lossless image compression based on the concept of psychovisual threshold. This paper proposes a practical image coding on large two-dimensional discrete cosine transform (DCT). A psychovisual model is presented following the psychoacoustic quite threshold as a just noticeable difference instead of a quantization table. An experimental result on large rectangular images shall be presented. This framework can easily produce higher quality images at a competitive compression rate in addition to an extended adaptive JPEG compression standard.
\end{abstract}

Keyword: Discrete cosine transform; Image coding; Just noticeable difference; Psychovisual threshold 